\title{
Vacuum method for creation of liquid crystal orienting microrelief
}

\author{
Yu. Kolomzarov, P. Oleksenko, V. Sorokin, P. Tytarenko, R. Zelinskyy \\ V.Lashkaryov Institute of Semiconductor Physics, NAS Ukraine, 41, prospect Nauki, 03028 Kyiv, Ukraine
}

\begin{abstract}
A mechanism for creation of microrelief surface anisotropy of amorphous films oxides materials which are obtained by oblique reactive cathode sputtering method is described. The influence of technological parameters of sputtering on the LC orienting parameters is investigated. The dependencies of the target material, angle of material emission and reemission processes under the substrate negative ion treatment is shown. The application of oblique reactive cathode sputtering method for creation of LCD with differ-ent size is demonstrated.
\end{abstract}

Keywords: liquid crystal, microrelief, reactive cathode sputtering.

Paper received 11.03.03; accepted for publication 11.12.03.

\section{Introduction}

Scientific-and-technical reviews devoted to orientational effects at the interface "liquid crystal (LC) - solid", methods of preparing various types of orientants [1-4] as well as to problems of interrelations be-tween physical properties of surfaces with electrooptical parameters of devices based on LCs [5-7] allow critical analysis of orientational methods, when taking into account currently industrial requirements. Nowadays, in industry, the technological orientation process is realized using both vacuum technologies of orienting film deposition [8] and vacuumless ones based, for example, on the specific mechanical rub-bing of organic films [9]. In this case, vacuum methods have some limitations as to the possibility to automate the process of orienting film deposition onto large substrate areas, which are related to specific-ity of thin film vacuum deposition methods. For vacuumless methods, the main problem is to control anchoring energy and orientation parameters of $\mathrm{LC}$, in particular, pre-title angles of LC molecules. Wide perspectives can be provided by the effect of induced anisotropy of microrelief on the orienting surface when exposing it by polarized light [10-12]. This method has no deficiencies inherent to the method of the mechanical rubbing and enables to effectively control orientation parameters. However, before its wide application in practice, it is necessary to solve a set of problems associated with thermal stability, reproducibility of orienting films and reliability.
One of the vacuum methods of microrelief creation is the ion beam alignment method based on polyimide surface bombardment by Ar ion beam [13,14]. But this method requires prior coating on the substrate the polyimide layer by a nonvacuum method.

In this work, we have represented investigation results of the new vacuum LC alignment method based on oblique reactive cathode sputtering (RCS) $[15,16]$.

\section{Experimental setup}

Our setup for deposition of orienting films by using the method of reactive cathode sputtering is shown in Fig. 1. A special water-cooled cathode unit 1 was mounted at the top part of VUP-5 installation jar.

As target material, we used wafers of silicon, germanium, nickel, tantalum, titanium, aluminum and in-dium that possess a good thermal contact with cathode plane. The total area of the sputtered target was approximately $50 \mathrm{~cm}^{2}$. Sputtering of targets was carried out in atmosphere of argon-oxygen mixture. Sub-strates were mounted in the special holder enabling to change the slope angle between normal to plane of the target and substrates within the range 0 to $90^{\circ}$. The discharge voltage was varied from 1 to $5 \mathrm{kV}$.

Deposition of orienting layers was performed using the pressure of operating gas mixture withing the range $6 \times 10^{-2}$ to $8 \times 10^{-3}$ Torr. The LC cells were assembled by standard method and filled by LC cyanobyphenyl type. The alignment of two glass plates before assembling was 


\section{Yu. Kolomzarov et al.: Vacuum method for creation of liquid crystal orienting ...}

provided in such manner to realize homogeneous or twist orientation of LC in the cell. The quality of homogeneous as well as twist orienta-tions was estimated using methods of polarization microscopy. The parameter of twist orientation $\mathrm{kt}$ was determined via the following expression

$$
k_{t}=\log \frac{I_{0}}{I_{t}}
$$

where $I_{0}$ and $I_{t}$ are the values of light flow transmitting through the cell when polarizers are parallel and the cell is not filled with LC and filled with the twist orientation one, respectively.

\section{Results and discussion}

Analysis of physical processes taking place under preparation of thin films by thermal sputtering and RCS shows that there is no direct similarity between them. While using RCS, the structure of the deposited film is determined by two main factors: deposition of substance onto the substrate and reemission of this substance by negative ions bombardment. Contribution of each factor is determined by plasma beam parameters. The bombardment by negative ions takes place just under the angle $\alpha$ relatively to the substrate surface, while condensation angles of sputtered substance have larger infinity through its collisions with gas ions and atoms as well as due to the fact that cathode dimensions are considerably larger than substrate projections onto the cathode. Being based on numerous experiments, we ascertained that the infinity in condensation angles for orienting substance can be essentially decreased if using the screen with a slot of the rectangular shape placed under the target at the distance 3 to $5 \mathrm{~mm}$, which enables to form the narrow plasma torch (Fig. 1).

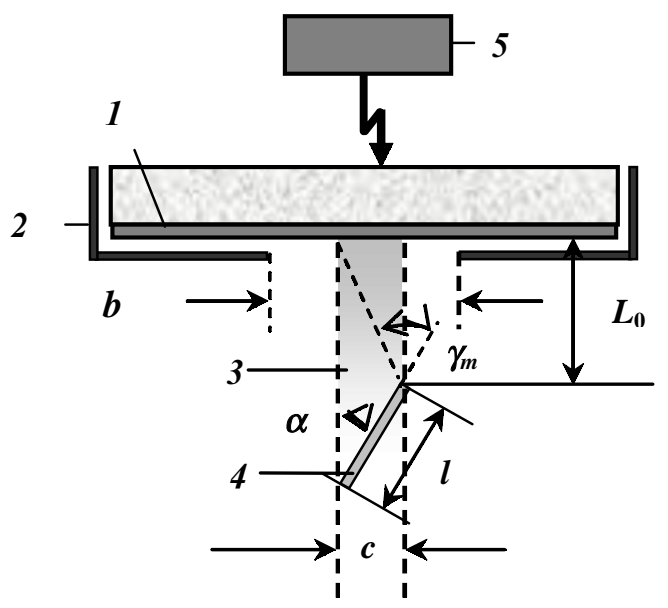

Fig. 1. Schematic of the cathode unit for deposition of orienting layers. 1 - cathode, 2 - screen, 3 - plasma torch, 4 - substrate, 5 - high voltage supply.
The symmetry axis of the slot should coincide exactly with the target center and that of the substrate holder. As it follows from Fig. 1, the condensation angles of sputtered substance will be limited by the well determined condensation angle $\gamma_{\max }$, while substrates will be treated in the plasma torch with the width $\mathrm{c}$, and $\mathrm{c}$ being always less than the slot width $b$. It is conditioned by squeezing the plasma torch by the screen, as it is placed within the range of dark cathode space.

It was ascertained that the orientation worsened with exceeding the substance maximal condensation angle. The peak value of the allowed substance condensation angle can be defined in the following manner:

$\gamma_{\max }=\alpha+\operatorname{arctg} \frac{c}{L_{0}}$

where $\alpha$ is the angle of the substrate slope relatively to the normal to cathode surface, $c$ is the width of the plasma torch, $L_{0}$ is the minimum allowed distance from the cathode to the upper substrate edge (the length of the dark cathode space).

Changing the plasma torch width, one can change the substance condensation angle and control, in this manner, the orientation parameters of LC films. It is experimentally found that the plasma torch width $\mathrm{c}$ is the function of at least four technological parameters, namely: the cathode voltage $U_{k}$, gas pressure in the operating mixture $p$, oxygen concentration in this mixture $k_{0}$ and the screen slot width $b$.

The deposition scheme shown in Fig. 1 possesses one essential deficiency - a low package density for substrates under the cathode and, as a consequence, a low productivity of this technological facility. For instance, if considering the substrates with sizes $25 \times 15 \mathrm{~mm}^{2}$ under given technological regimes for orienting layer deposition that provides the high quality of LC orientation, then the necessary plasma torch width $c=8 \mathrm{~mm}$ is reached with the slot width $b=28 \mathrm{~mm}$, i.e., practically $60 \%$ of the cathode area is used inefficiently.

To avoid this deficiency, we developed the second way. Fig. 2 shows the scheme of appliance capable to realize the developed way of orienting coating deposition, and Fig. 3 represents the deposition scheme used in this way. In accord with Fig. 2, the screen is designed in the form of sections with the height and length limited by the cathode dimensions.

The separation between sections was chosen as equal to $l \sin \alpha$, where $l$ is the substrate length. The distance $L$ between the screen 2 and cathode $l$ was chosen in such a manner that it was larger than or equal to the length of the dark cathode space in this glow discharge. The substrates 3 were mounted into the holder 4 in such a manner that their plane made the angle $\alpha$ relatively to the plasma torch direction.

To determine the screen section height, we shall use Eq. (2) and Fig. 2. Taking into account that in the first way $c=8 \mathrm{~mm} L_{0}=34 \mathrm{~mm}$, let us deduce the maximum substance condensation angle for this sub-strate: 


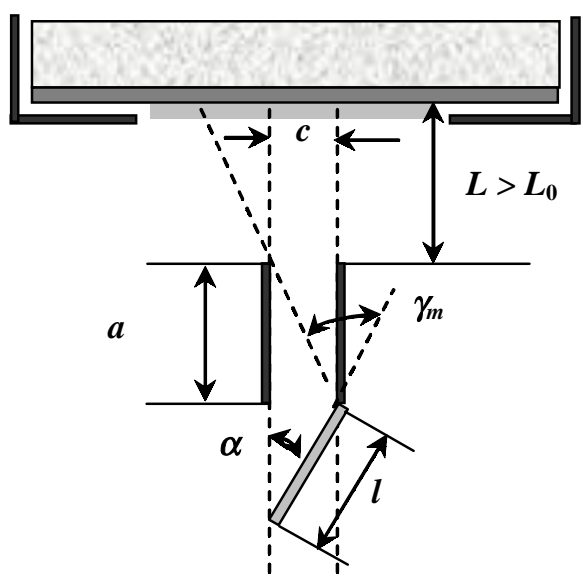

Fig. 2. Scheme of the facility for deposition of orienting layers, which is suitable to optimize substance condensation angles.

$$
\gamma_{\text {max }}=\alpha+13.2^{\circ}
$$

On the other hand, it follows from Fig. 2 that the expression for the maximum substance condensation angle in the second way of orienting coating deposition has the following form:

$\gamma_{\max }=\alpha+\operatorname{arctg} \frac{l \sin \alpha}{a}$

Having jointly solved Eqs (3) and (4), we get

$a=4.3 l \sin \alpha$

Validity of the equality (5) is one of the necessary conditions for the offered way to be realized.

In this case, with decreasing the screen section height, $\gamma_{\max }$ is increased. As a consequence, LC orientation is worsened. Therefore, in a common case, when realizing the offered method, the value a should be cho-sen using the following inequality

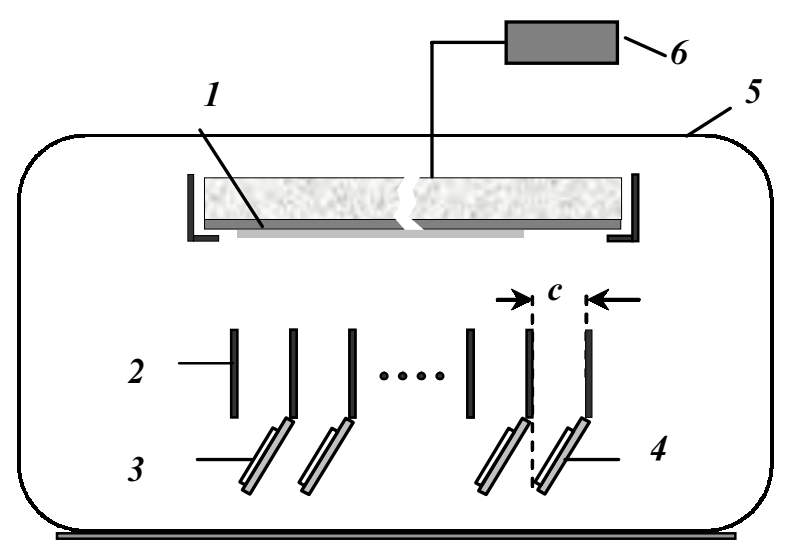

Fig. 3. Scheme of an under-jar setup in the industrial unit for deposition of orienting structures: 1 - cathode, 2 - screen system, 3 - substrate, 4 - substrate holder, 5 - jar, 6 - high voltage supply. $a \geq 4.3 l \sin \alpha$

The second necessary condition to realize this way is the necessity to place the screen at the distance $L$ from the cathode, which is large or equal to the length of the glow discharge dark cathode space $L_{0}$ :

$$
L \geq L_{0}
$$

As compared with the first way, the productivity of the installation is approximately three-fold increased when deposing these orienting coatings.

To solve the tasks of orienting layer deposition onto substrates with a large area, we have developed the scheme of a cathode unit enabling to scan the substrate in the plasma torch (Fig. 4). This modification of the cathode unit provides formation of a narrow plasma torch with $5 . .8 \mathrm{~mm}$ width and $150 \mathrm{~mm}$ length as well as the possibility of substrate alternate motion with the velocity 0.5 to $5 \mathrm{~mm} / \mathrm{s}$, the substrate being mounted at the angle $\alpha=5$ $30^{\circ}$.

This configuration provides the possibility to obtain an equalthickness coating over the whole area of the substrate with large sizes $\left(150 \times 150 \mathrm{~mm}^{2}\right)$ as well as the potential to simultaneously deposit the orienting coatings onto large quantity of substrates with a small area. Our investigations of orienting properties in-herent to oxides films of indium, aluminum, germanium, silicon, tantalum, titanium showed that $\mathrm{SiO}_{\mathrm{x}}$ and $\mathrm{GeO}_{\mathrm{x}}$ layers, as to the above properties, are capable to compete with thermally deposited ones. Experiments showed that the quality of twist orientation promoted by $\mathrm{SiO}_{\mathrm{x}}$ and $\mathrm{GeO}_{\mathrm{x}}$ films is $K_{t} 2.5$, which meets well the requirements to LC displays. However, $\mathrm{SiO}_{\mathrm{x}}$ orientants are more transparent and less hydrated. Therefore, these were preferred in our investigations. The performed study showed that the orientation quality depends on a great amount of technological parameters in the course of $\mathrm{SiO}_{\mathrm{x}}$ layer deposi-

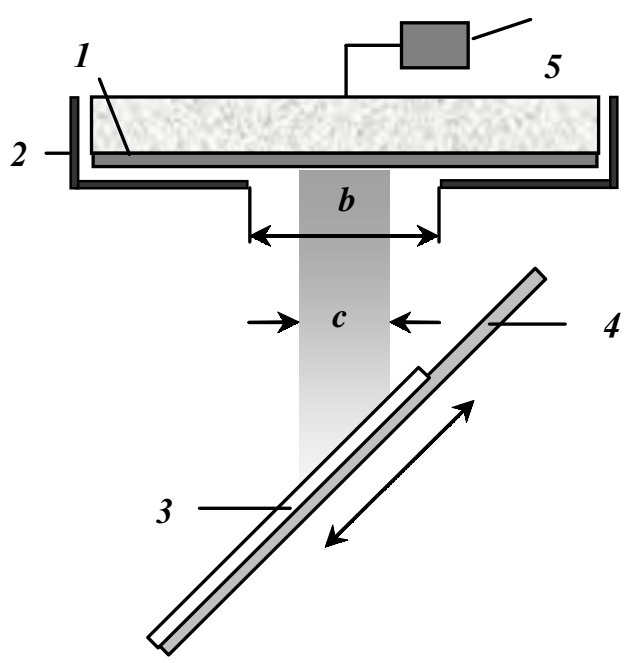

Fig. 4. Scheme of the cathode unit with configuration enabling to scan substrates in the course of orienting layer deposition: 1 cathode, 2 - screen, 3 - substrate, 4 - scanning system, 5-high voltage supply.

SQO, 6(4), 2003 


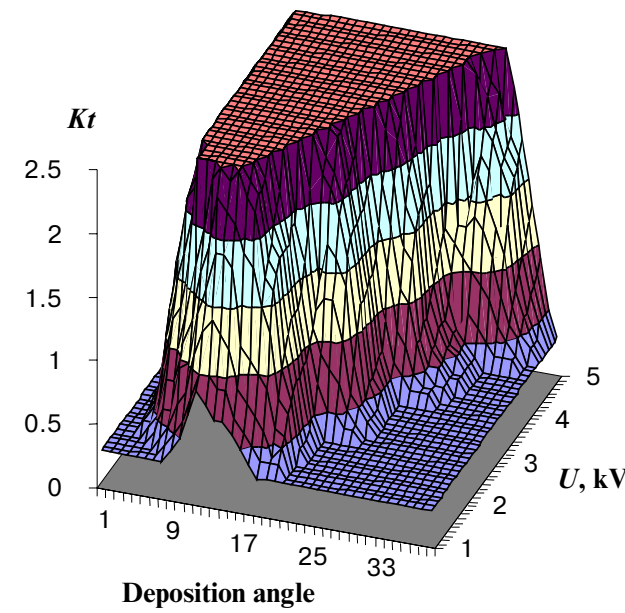

Fig. 5. Nomogram for determination of the technological parameters.

tion (the cathode voltage, discharge current density, operating gas pressure, oxygen concentration in an operating mixture, angle and time of deposition, etc.).

The dependencies of parameter of twist orientation $K_{t}$ from the deposition angle and cathode voltage $U_{c}$ is shown in Fig. 5 as 3D representation. This characteristic was plotted on the base of fair amount of tech-nological experimental data. Upper flat zone of the graphic corresponds the technological parameters which allow to rich $K_{t}>2,5$. For example, at the given deposition angle $\alpha=$ $=15^{\circ}$ and the voltage $U_{c}=3 \mathrm{kV}$, the deposition process should be carried out at the current density $j=$ $=3.2 \mathrm{~mA} / \mathrm{cm}^{2}$. Minimal current den-sity of plasma was calculated from experimental formula $j=4,73-0,5 U_{c}$. The voltage or discharge current density increase results only in growing $K_{t}$.

The experimental investigations of the developed method applicability to deposing oxides of such materials as silicon, germanium, aluminum, tantalum, indium etc. showed the possibility to form oxide structures of above materials capable to orient molecules of nematic LC in the direction coinciding with that of ion bombardment in the course of material deposition. The substrate plane should be oriented at the definite angle to the cathode plane normal.

The study of orienting layer capabilities and optical properties of these layers showed that formation of the orienting structure is determined by two main processes: emission (the sputtered substance is deposited onto a sustrate after its oxidation in plasma) and re-emission (the deposited layer is under bombardment by negative ions).

The study of surface morphology by Atomic Force Microscope showed absence of open orienting texture for the CRS substrate (Fig. 6b) in comparison with rubbing polyimid substrate (Fig. $6 a$ ). The same result showed AFM investigation of the orienting surfases obtained by
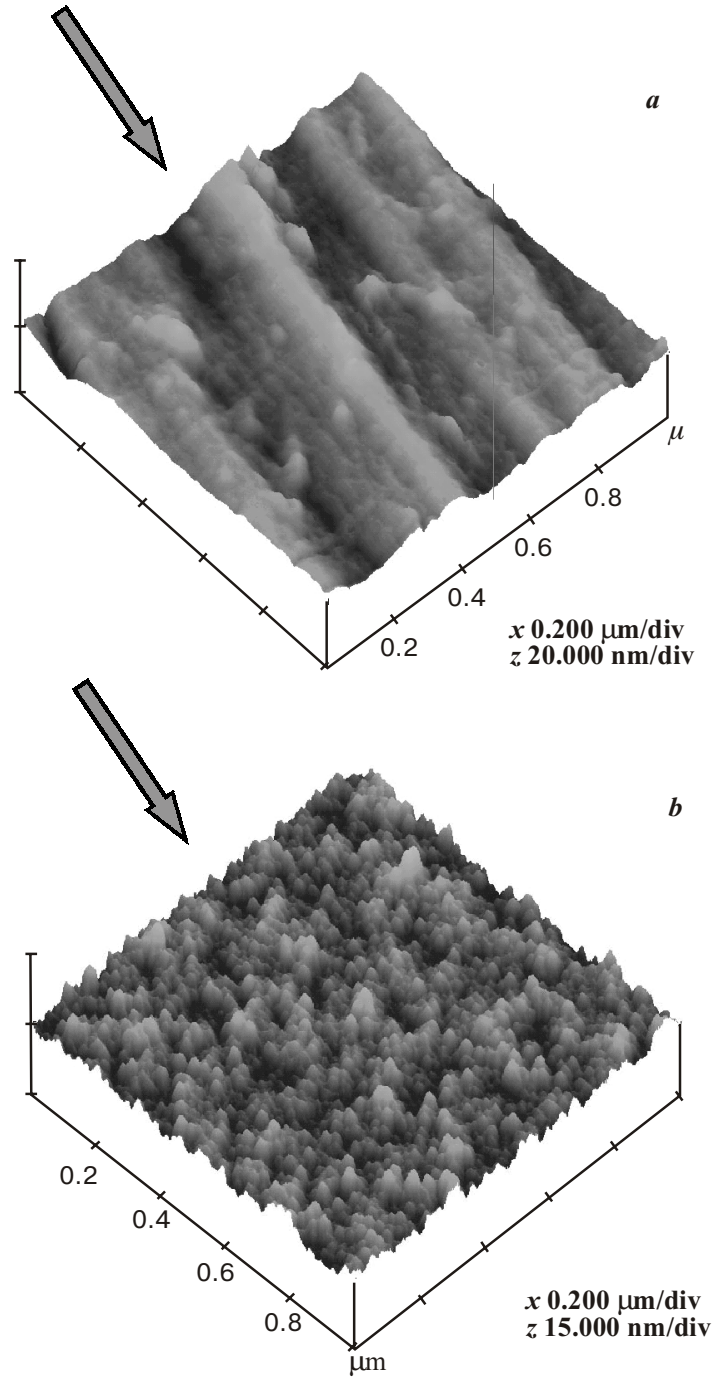

Fig. 6. Morphology of orientating textures obtained by rubbing (a) and CRS method (b).

the ion beam alignment method [14]. For final ascertaining the physical mechanism of orientation by CRS method, additional investigation of surface morphology and physicochemical properties of the system LC-orientating surface are neccessary.

\section{Conclusions}

The experimental investigations of the developed method applicability to deposing oxides of such materi-als as silicon, germanium, aluminium, tantalum, indium etc. showed the possibility to form oxide struc-tures of above materials capable to orient molecules of nematic LCs in the direction coinciding with that of ion bombardment in the course of material deposition. The substrate plane should be oriented at the definite angle to the cathode plane normal. 


\section{Yu. Kolomzarov et al.: Vacuum method for creation of liquid crystal orienting ...}

The orientation properties of substrates created by CRS method showed that formation of the orienting structure is determined by two main processes: emission (the sputtered substance is deposited onto a sustrate after its oxidation in plasma) and reemission (the deposited layer is under bombardment by negative ions). The offered method opens a direct way to create ecologically pure technology of LC dis-plays without using harmful chemical compounds.

\section{References}

1. E.S. Luk'yanchenko, V.A. Kozunov, V.I. Grigos, Orientation of nematic liquid crystals // Uspekhi khimii, 54(4), pp. 214238 (1985) (in Russian).

2. W.J.A. Ctoosens, Bulk interfacial and anchoring of liquid crystals // Mol. Cryst.Liq.Crys., 124.(1-4), pp.305-319 (1985).

3. V.G.Chigrinov, T.V. Korkishko, Role of surface in electrooptics of liquid crystals // Abstracts of the Fifth All-Union Conference "Liquid Crystals and Their Practical Application” 2(1-2) pp. 6-51. Ivanovo, (1985) (in Russian).

4. Zh. Konyar Orientation of nematic liquid crystals and their mixtures. "Universitetskoye", Minsk, 1986 (in Russian).

5. V.G. Chigrinov Orientational effects in electric and magnetic fields // Kristallographiya, 27(2), pp. 404-430 (1982) (in Russian).

6. V.A. Kozunov, V.G. Chigrinov, Electrooptics of the twist effect in liquid crystals // Obzory po elektronnoi tekhnike. Ser. 3. Mikroelektronika 8, (1988) (in Russian).

7. D. Berreman, Alignment of liquid crystals by groved surfaces // Mol.Crys.Liq.Crys. 23(3-4), p.215-231 (1973).
8. O.V. Gorbunov, S.P. Kurchatkin, A.A. Mukhayev et al. Orientation of liquid crystals using germanium monooxide // Izvestiya AN SSSR. Ser. Neorganich. Mater. 19(3) p.p. 467471 (1983) (in Russian).

9. H.Tabira, T.Inoue, Y.Yahagi, H.Imayama, M.Morimoto. Precision rubbing supported by fine process analysis// J. of the SID.10(4), p.329-337 (2002).

10. D. Fedorenko, E. Ouskova, Yu.Reznikov, L.Su, S.Shiyanovskii, O. Kuksenok, J. Wesi, O.Francescangeli, F.Simoni, Hidden Photoalignment of Liquid Crystals in isotropic Phase // Phys. Rev. E. 63, 021701. p.p.5. (2001)

11. D.Andrienko, D.Fedorenko, O.Uskova, V.Reshetnyak, Yu.Reznikov, S.Slussarenko, D.Voloshchenko, O. Lavrentovich, Light-induced Alignment and Reorientation Effects in Liquid Crystals Doped with Azo-dyes // Ukr. Journ. of Phys. 44(1-2), p.p. 149-157 (1999)

12. D.Fedorenko, E.Ouskova, Yu.Reznikov, Reshetnyak V. S.Shiyanovskii, O.Francescangeli, F.Simoni, Properties of Bulk-mediated Photoalignment of Doped Liquid Crystal // Mol. Cryst. Liq. Cryst. 359, p.p..137-145 (2001)

13. D.J.Kang, J.S.Gwag, J.W.Kang, T-H.Yoon, J.C.Kim, H.Kim, S.J.Cho, Out-of-Plane Alignment Properties of Nematic Liquid Crystals on Polyimide Surface Bombarded by Ar Ion Beam //SID 03 DIGEST, pp. 624-627(2003)

14. J.S.Gwag, K-H.Park, D.J.Kang, C.G.Jhun, H. Kim, T.H.Yoon, J.C.Kim, Polyimide Surface Bombarded with Ar Atomic Beam// Jpn. J. Appl. Phys. 42, pp.L468-L471 (2003)

15. Sorokin V.M., Oleksenko P.F., Ovsyannikov V.D. The method of orienting film preparation. Au-thor's certificate 1009109.

16. Anoshkin A.V., Zelinskyy R.Ya., Kuz'min N.G., Sevostyanov V.P., Sorokin V.M. The facility for deposition of orienting films. Author's certificate 1492736. 